\title{
APLICAÇÃO DE UMA CURVA DE GANHO DE PESO PARA GESTANTES
}

Arnaldo Augusto Franco de Siqueira* Cyro Ciari Junior *

Iara Lucia Brayner Mattos*

Keiko Ogura Buralli *

Malaquias Baptista Filho**

Néia Schor *

Pedro Augusto Marcondes de Almeida *

Ana Cristina d'Andretta Tanaka*

RSPU-B/361

Siqueira, A. A. F. de et al. Aplicação de uma curva de ganho de peso para gestantes. Rev. Saúde públ., S. Palllo, 11:288-93, 1977.

RESUMO: Com a finalidade de estudar a influência do estado nutricional materno sobre o peso do recém-nascido, foi aplicado em 460 gestantes inscritas no Centro de Saúde Geraldo de Paula Souza o método antropométrico para o diagnóstico do estado nutricional de uma populaçấo de gestantes, pois este método é de fácil aplicação e não exige pessoal especializado. Parece existir uma relação positiva entre o peso pré-gestacional, o ganho de peso da gestante durante a gravidez, e o peso ao nascer do concepto, bem como a idade gestacional. Observout-se ainda que houve uma diferença de 296,7 gramas a mais no peso dos recém-nascidos de gestante com peso pré-gestacional adequado, que no grupo de gestante com peso pré-gestacional insuficiente. Em trabalhos anteriores descreveu-se a necessidade de se utilizar curvas ponderais para o diagnóstico do estado nutricional materno: a curva que ora se apresenta é mais simples que outras já descritas anteriormente. No momento está sendo testada para se avaliar a sua eficácia.

UNITERMOS: Gestantes, estado nutricional. Prematuros. Recém-nascidos, estado nutricional. Peso ao nascer. Assistência pré-natal.

Nos últimos anos tem havido um interesse sempre crescente pela nutrição materna e fetal. Essa preocupação se deve ao fato de a desnutrição intra-uterina ser um dos principais fatores responsáveis pelo baixo peso do recém-nascido, bem como pela elevada mortalidade neonatal que se verifica em regiões em desenvolvimento, como é o caso de São Paulo ${ }^{3}$.

Dentre as várias técnicas preconizadas para o diagnóstico do estado nutricional de uma população, parece fora de dúvida que os métodos antropométricos devam ser os preferidos, pelo seu baixo custo e simplici-

* Do Departamento de Saúde Materno-Infantil da Faculdade de Saúde Pública da USP - Av. Dr. Arnaldo, 715 - São Paulo, SP - Brasil.

* Da Escola Paulista de Medicina - Rua Botucatu, 720 - São Paulo, SP - Brasil. 
SIQUEIRA, A. A. F. de et al. - Aplicação de uma curva de ganho de peso para gestantes. Rev. Saúde públ., S. Paulo, 11:288-93, 1977.

dade de aplicação, desde que permitam identificar as gestantes desnutridas ou que corram um risco maior de ter filhos de baixo peso.

Em trabalhos anteriores $2,4, .5$ foram construídas e aplicadas curvas de ganho de peso de gestantes, qi'c se mostraram úteis para esse propósito. No entanto, o fato de serem seis curvas dificulta a sua aplicação. Além disso, por serem aplicadas, por exemplo, em mulheres de estatura 1,50 a 1,54 , é mais provável que os pesos de mulheres de menor estatura estejam em posição inferior na curva. Havia, então, necessidade de uma curva para cada estatura.

O Instituto de Nutrição de Centro América e Panamá (INCAP) utiliza uma curva que, a partir da relação peso-altura, classifica as mulheres (não gestantes) em: peso normal, peso baixo e peso acentuado. Uma segunda curva qualifica o ganho de peso da gestante em: insuficiente, adequado ou acentuado segundo esteja abaixo, dentro ou acima de uma faixa de valores normais (Figura).

O objetivo deste trabalho foi verificar se essas curvas, que foram por nós reconstruidas com base em trabalhos anteriores ${ }^{2.4 .5}$, podem ou não ser utilizadas para diagnosticar o estado nutricional de gestantes medido a partir da influência sobre o peso do recém-nascido.

\section{MATERIAL E MÉTODOS}

Foran utilizados os dados referentes a 486 gestantes sadias atendidas no Serviço de Pré-Natal da Faculdade de Saúde Pública, dados esses já referidos em outro trabalho:

As curvas foram aplicadas a 460 dos 486 casos, pois foram desprezados os casos de gestantes que só apresentavam 3 tomadas de peso durante a gestação.

Para que o peso da gestante na primeira consulta pudesse ser utilizado, era sempre subtraído o peso correspondente do ganho médio de peso devido à própria existência da gravidez.
Assim, para gestação até a $8^{n}$ semana 0 peso atual da mulher era considerado o mesmo peso pré-concepcional; para gestaçoes de 11 semanas, foi subtraído $0,5 \mathrm{~kg}$ do peso das gestantes; para gestações de 15 semanas foi subtraido $1,3 \mathrm{~kg}$ e assim por diante.

As gestantes foram, assim, classificadas como sendo de peso inicial insuficiente, adequado ou acentuado.

A seguir, passou-se ao acompanhamento do ganho de peso da gestante. Considerouse que o ganho de peso é linear no $2^{\circ}$ e $3^{\circ}$ trimestre e que, no fim da gestação (42 semanas), a mulher deve ganhar 9 a 13 quilos. Assim, no último peso medido da gestante, era feita nova classificação em insuficiente, adeyuado ou acentuado.

\section{RESULTADOS E DISCUSSĀO}

Podemos dizer que a aplicação dessa curva é muito simples, exigindo apenas um treinamento sumário para o seu uso adeyuado.

Na Tabela 1, verifica-se que os filhos de mulheres de peso inicial (pré-concepcional) insuficiente apresentam um peso médio ao nascer inferior ao das demais. Além disso, há uma elevada proporção de recém-nascidos de baixo peso (crianças de peso igual ou inferior a $2500 \mathrm{~g}$ ) e de peso insuficiente (crianças de peso superior a $2500 \mathrm{~g}$ mas igual ou inferior a $3000 \mathrm{~g}$ ). Essas proporções somadas abrangem a mais da metade dos casos $(51,7 \%)$.

Para os filhos de mulheres de peso inicial adequado o peso médio ao nascer foi $209 \mathrm{~g}$ maior, e a frequiência de recém-nascidos de baixo peso foi de $7,8 \%$; os de peso insuficiente foram $24,9 \%$ o que perfaz um total de $32,7 \%$, ou seja, menos de $1 / 3$.

O mesmo ocorre para os filhos de mulheres de peso inicial acentuado, em que há apenas $6,3 \%$ de recém-nascidos de baixo peso e $17,1 \%$ de peso insuficiente, num total de $23,4 \%$, ou seja, menos de $1 / 4$. 
SIQUEIRA, A. A. F. de et al. - Aplicação de uma curva de ganho de peso para gestantes. Rev. Saúde públ., S. Paulo, 11:288-93, 1977.
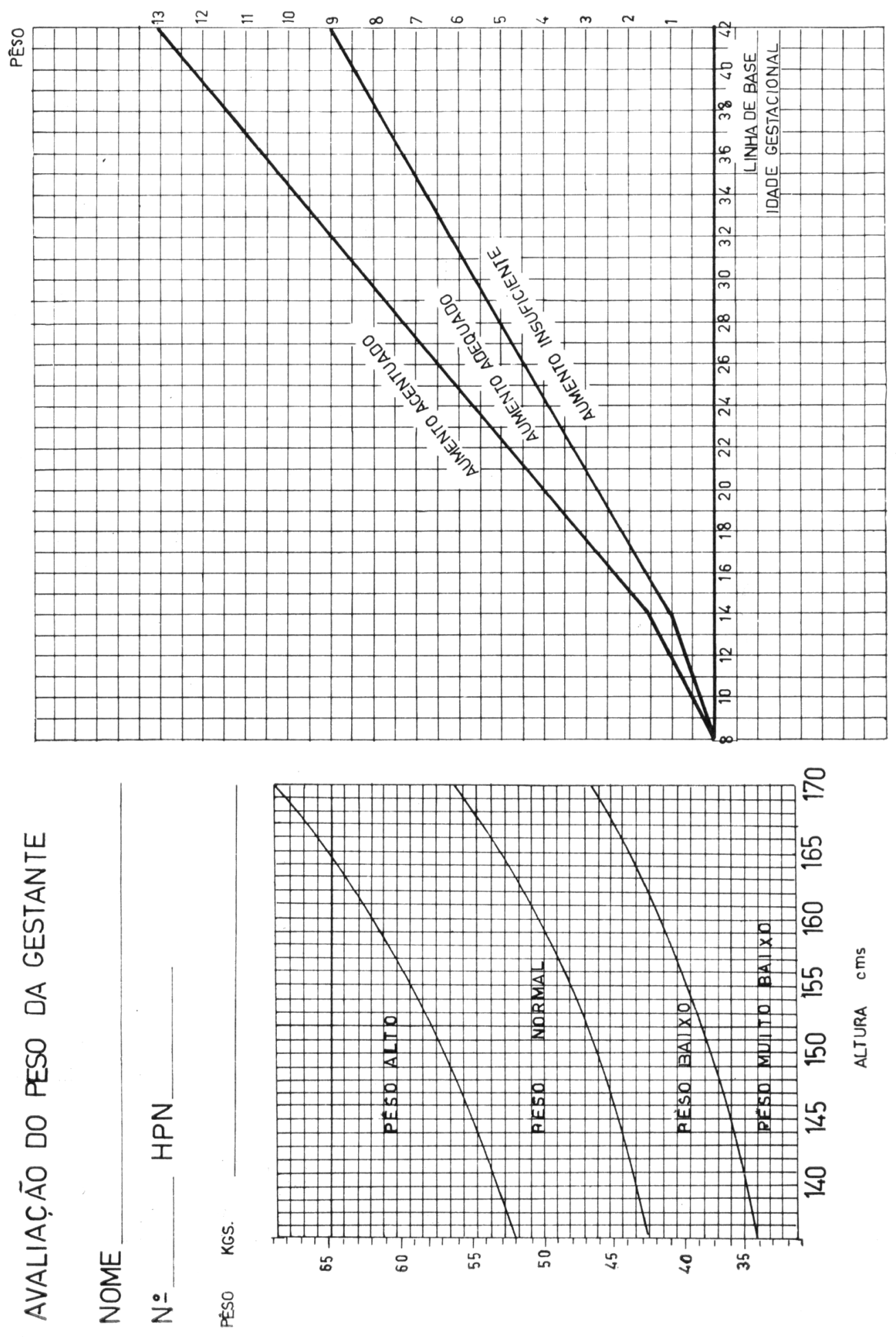
SIQUEIRA, A. A. F. de et al. - Aplicação de uma curra de ganho de peso para gestantes. Rer. Saúde puibl., S. Paulo. $11: 288-93,1977$.

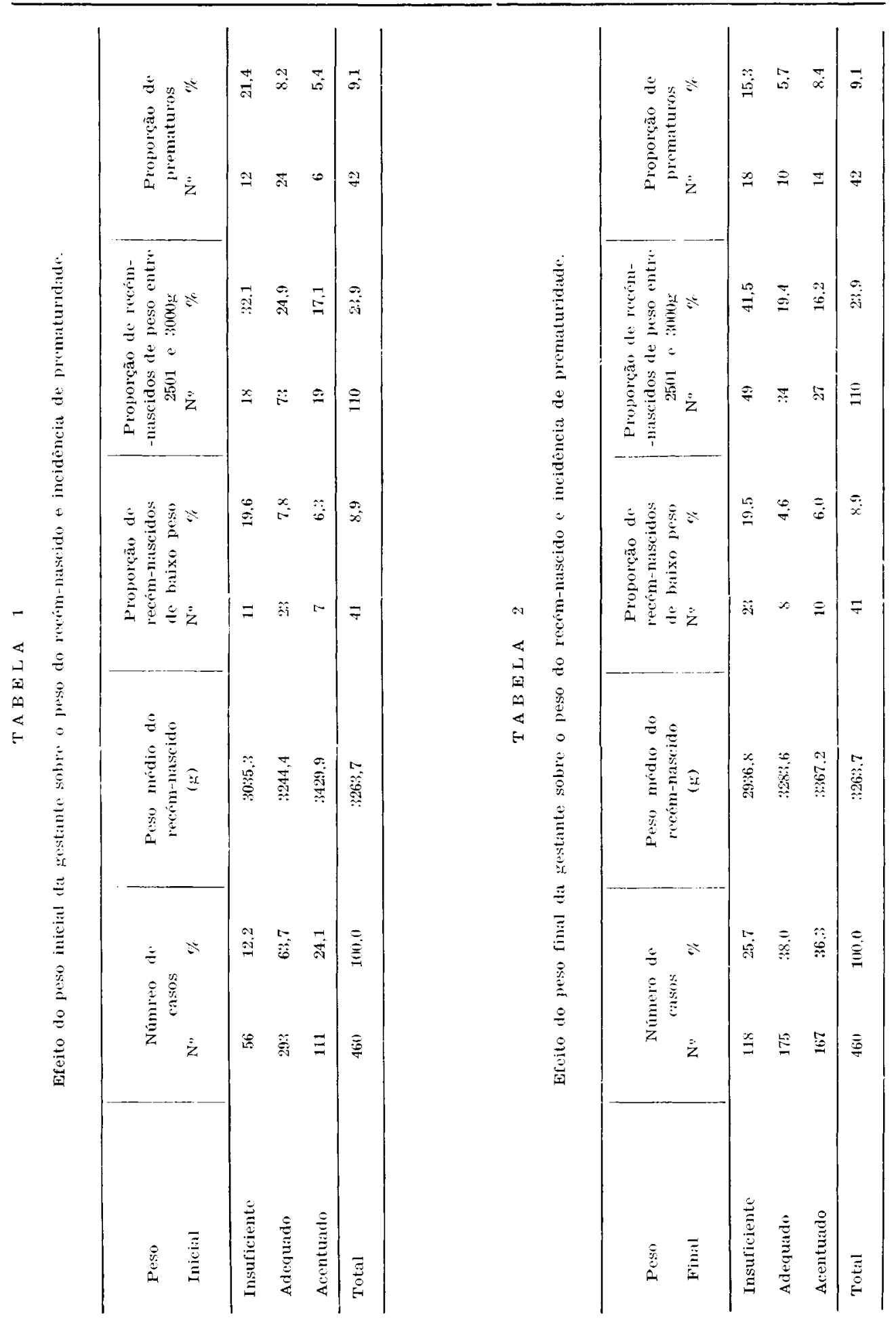


SIQUEIRA, A. A. F. de et al. - Aplicação de uma curra de ganho de peso para gestantes.

Rev. Saúde pübl., S. Paulo, 11:288-93, 1977.

Além desa proporção maior de recémnascidos de baixo peso e peso insuficiente, mais de $20 \%(21,4 \%)$ dos filhos le mulheres de peso insuficiente foram prematuros, isto é, nasceram de gestaŗóes de 37 semanas ou menos. Este fato ja foi por nós descrito $=$

Fica claro, assin, que o peso inicial da gestante guarda umı relação direta com () peso do recém-nascido.

A Tabela 2 permite analisar o comportamento do peso da gestante durante a gravidez, ou seja, classificar o ganho de peso em insuficiente, adequado ou acentuado, conforme o peso da gestante no fim da gravidez esteja abaixo, dentro ou acima de uma faixa de valores normais.

O que se pretende, quando a curva é aplicada prospectivamente, $\dot{e}$ que a mulher tenha um ganho de peso adequado ao seu peso inicial; assim, se a primeira pesagem a gestante é consideralda como de peso insuficiente, deverá ganhar os a a 13 quilos de uma gestação normal e mais a diferença entre o seu peso e u peso (nnsiderad(s) normal para a sua cstatura. Da mesma forma, a gestante de peso inicial acentuado deverá ganhar os 9 a 13 quilos menos 0 total de quilos que apresente a mais do seu peso normal.

Neste trabalho, como a curva foi aplicada retrospectivamente, não foi possivel verificar o efeito de sua utilização sobre o peso do concepto pois não houve, como é óbvio, orientação para quc as gestantes ganhassem mais ou menos peso de acordo com o seu peso inicial.

Mesmo assim, os fi'hos de nulheres que apresentavam peso final insuficiente tinham peso médio muite menor que os demais grupos $(2.936,8 \mathrm{~g})$. A proporção de recémnascidos de baixo peso, peso insuficiente $e$ prematuros era muito elevada também, em relação aos demais grupos (Tabela 2 ).

Interessante é notar que quando a gestante tem pesos pré-concepcional e final insufi- cientes, a incidência de prematuridade chega a $28,2 \%$ dos casos. Quando a gestante de peso pré-concepcional insuficiente tem um ganho de peso que a leva a un peso final adequado ou até mesmo acentuado, a incis dência de prematuridade é de $6,7 \%$, valor considerado normal.

Outro resultado interessante é dado pelo peso médio ao nascer de filhos de gestantes que tiveram ganho de peso adequado, que foi 296,7 gramas maior que o de grupo de mães com ganho de peso insuficiente.

Em rista desses resultados iniciais, acreditamos que essa curva, desenvolvida no Departanento de Saúde Materno-Infantil da Faculdade de Saúde Pública da USP, a partir de uma outra que foi usada pelo INCAP, merece ser mais amplamente testada e parece ser um bom instrumento de medida em Nutrição Materna.

C O N L L U O ES

1. A curva por nós desenvolvida é de fácil aplicação não exigindo formação profissional.

2. Os resultados obtidos com a aplica(ção desta curva foram semelhantes aus observados em trabalhos anteriores.

3. () peso pré-gestacional determina en grande parte o peso ao nascer do concepto.

4. O ganho de peso da gestante, sendo aclequado ao peso pré-gestacional, influi positivamente no peso ao nascer.

5. A incidencia de prematuridade é elevada $(28,2 \%)$ no grupo de gestantes que apresentam peso pré-concepcional e final insuficientes, em comparação com os outros grupos, cuja freqüência de prematuridade está situada em níveis considerados normais.

6. O peso médio do recém-nascido do grupo de gestantes com ganho de peso gestacional adequado foi maior em $296,7 \mathrm{~g}$ em relação ao grupo de gestante com ganho de peso gestacional insuficiente. 
SIQUEIRA, A. A. F. de et al. - Aplicação de uma curva de ganho de peso para gestantes. Rev. Saúde públ., S. Paulo, 11:288-93, 1977.

RSPU-B 361

SIQUeIRA, A. A. F. de et al. [Application of a weight gain curve for pregnant women]. Rev. Saúde públ., S. Paulo, 11:288-93, 1977.

ABSTRACT: The authors studied the influence of maternal nutrition on birth weight using anthropometric techniques because these methods are of low cost, and do not need specialized manpower for their application. In the 460 pregnant women attended at a prenatal care service, there was a positive relationship between pre-gestational weight gain during pregnancy, birth weight and gestational age. Babies born from mothers with adequate pre-gestational weight were 296.7 grammes heavier than those of women with insufficient weight. Anthropometric curves for the measurement of maternal weight have been simplified and now this new curve is being tested.

UNITERMS: Pregnancy, nutritional status. Infant, premature. Infant newborn, nutritional status. Birth weight. Prenatal care.

\section{REFERENCIAS BIBLIOGRAFICAS}

1. CIARI Jr.. C. Curva ponderal de gestantes normais. Folha méd., 68:141-6. 1974.

2. SIQUeIrA, A. A. F, de et al. Influencia da altura e ganho de peso maternos e de idade gestacional sobre o peso do recém-nascido: estudo de très grupos de gestantos normais. Rer. Saúde puibl., S. Paulo, 9:331-42. 1975.

$\because$ SIQUEIRA, A. A. F. de Morialiade neo-natal e prematurdade. São Paù. 1974 [Monografia de mestrado -Faculdade de Saúde Pública da USP]
4. Siqueira. A, A. F. de et al. Utilização de uma curva de crescimento intrautemo corrigida para peso e altura maternos. Rev. Saúde pübl., S. Paulo, 9:215-20, 1975

5. SIQLEIRA, A. A. F. de et al. A utilização de uma curva ponderal de gestantes normais, no diagnóstico de desnutrição intra-uterina. R€v. Saúde públ., S. Paulo, 9: 495-506., 1975.

Recebido para publicação em 1.3/12/1976 Aprovado para publicaça a em 1\%/12/1976 